\title{
Symmetry breaking of two-dimensional time-periodic wakes
}

\author{
By H. M. B L A C K B UR N, ${ }^{1}$ F. M A R Q U E S ${ }^{2}$ \\ AND J. M. LOP E Z $\mathbf{Z}^{3}$ \\ ${ }^{1}$ CSIRO Manufacturing and Infrastructure Technology, P. O. Box 56, Highett, Vic. 3190, \\ Australia \\ ${ }^{2}$ Departament de Física Aplicada, Universitat Politècnica de Catalunya, 08034, Barcelona, \\ Spain \\ ${ }^{3}$ Department of Mathematics and Statistics, Arizona State University, Tempe, AZ 85287, USA
}

(Received 12 June 2004)

A number of two-dimensional time-periodic flows, for example the Kármán street wake of a symmetrical bluff body such as a circular cylinder, possess a spatio-temporal symmetry: a combination of evolution by half a period in time and a spatial reflection leaves the solution invariant. Floquet analyses for the stability of these flows to three-dimensional perturbations have in the past been based on the Poincaré map, without attempting to exploit the spatio-temporal symmetry. Here, Floquet analysis based on the half-periodflip map provides a comprehensive interpretation of the symmetry breaking bifurcations.

\section{Introduction}

When a system is invariant under the action of a group of symmetries, there can be far-reaching consequences on its bifurcations. When the symmetries are purely spatial in nature (e.g. reflections, translations, rotations), these consequences have been extensively studied (see, for example, Golubitsky \& Schaeffer 1985; Golubitsky, Stewart \& Schaeffer 1988; Crawford \& Knobloch 1991; Cross \& Hohenberg 1993; Chossat \& Iooss 1994; Iooss \& Adelmeyer 1998; Chossat \& Lauterbach 2000; Golubitsky \& Stewart 2002). The system may also be invariant to the action of spatio-temporal symmetries. These are spatial symmetries composed with temporal evolution. A classic example is the two-dimensional Kármán vortex street form of the wake of a circular cylinder. When snapshots of dye or hydrogen bubble visualisation of the wake are taken half a shedding period apart, these manifest an invariance corresponding to a reflection about the wake centreline (the action of the spatial reflection symmetry) together with a half period temporal evolution. Figure 1 shows computed locations of marker particles in the two-dimensional cylinder wake half a period apart, illustrating this spatio-temporal symmetry. This symmetry is an involution, i.e. applying it twice is the same as applying the identity operator, and so it is a spatio-temporal $Z_{2}$ symmetry.

The transition from two-dimensional to three-dimensional flow is of fundamental interest in fluid dynamics. Two-dimensional flows, like the Kármán vortex street and other bluff body wakes, are invariant in the spanwise direction to both translations $(S O(2)$ symmetry group) and to reflections $\left(Z_{2}\right.$ symmetry group), the combination generating the $O(2)$ symmetry group. The complete symmetry group of these flows is $Z_{2} \times O(2)$. The implications of $O(2)$ symmetry in fluid systems have been extensively studied, both when the instability breaking $O(2)$ symmetry (i.e. transition from two-dimensional to 


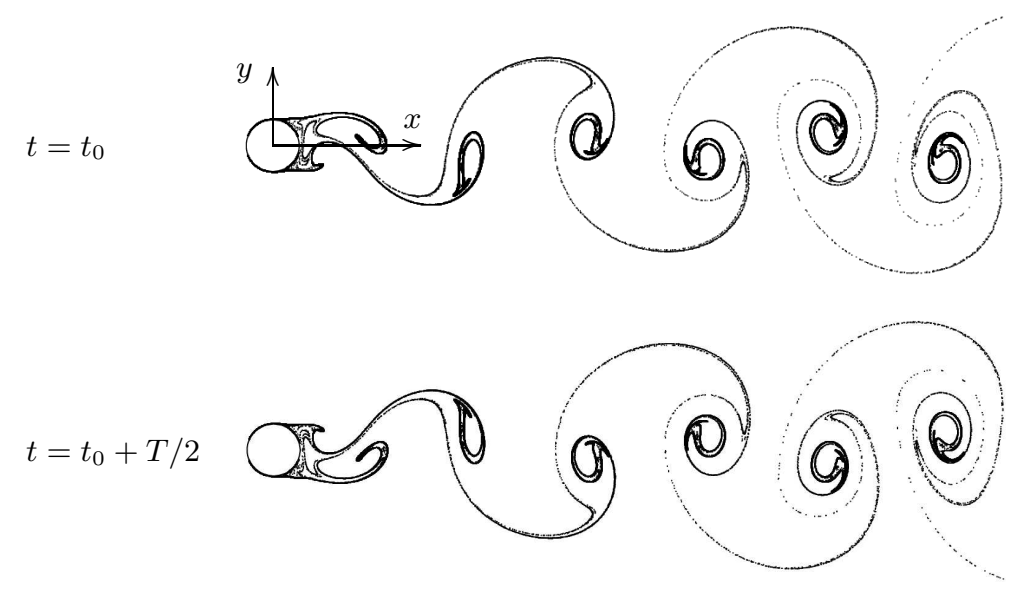

Figure 1. Computed locations of marker particles, illustrating the spatio-temporal symmetry of a two-dimensional circular cylinder wake for $R e=188.5$ at times $t_{0}$ and $t_{0}+T / 2\left(t_{0}\right.$ is arbitrary, $T$ is the Strouhal period).

three-dimensional) is due to a single real eigenvalue becoming positive (steady bifurcation) as well as when it is due to a pair of complex-conjugate eigenvalues gaining positive real part, leading to time-periodic flow (e.g. see the references cited above).

The types of symmetry breaking bifurcations to three-dimensional flow that a twodimensional flow can experience are completely determined by the symmetry group of the system, and not by the particulars of the physical mechanism responsible for the bifurcation. For example, spatial reflection symmetries (with a $Z_{2}$ symmetry group) are broken by pitchfork bifurcations in many flows, (e.g. Benjamin 1978a,b; Rucklidge 1997; Blohm \& Kuhlmann 2002), but may arise through different mechanisms, e.g. centrifugal, buoyancy or shear instability, in different situations. The point is that the symmetries of the system govern the types of possible bifurcations that may occur, as well as the symmetry properties of the bifurcating solutions themselves, an idea that is formalised in the equivariant branching lemma (see, for example Golubitsky, Stewart \& Schaeffer 1988; Chossat \& Lauterbach 2000).

The instabilities of two-dimensional time-periodic flows (e.g. the wake flows with spatio-temporal $Z_{2} \times O(2)$ symmetry) are governed by the Floquet multipliers whose moduli become greater than unity (Joseph 1976). In numerous wake flows, two distinct synchronous three-dimensional modes (i.e. with real Floquet multipliers) have been observed experimentally and computed as direct instabilities from the two-dimensional flow (e.g. Williamson 1988; Meiburg \& Lasheras 1988; Lasheras \& Meiburg 1990; Williamson 1996; Barkley \& Henderson 1996; Robichaux, Balachandar \& Vanka 1999; Barkley, Tuckerman \& Golubitsky 2000; Julien, Lasheras \& Chomaz 2003). In the various cases, the two modes are often differentiated by their spanwise wavelength, whether one or the other is the first to bifurcate from the two-dimensional state as a parameter (typically the Reynolds number) is varied, and to some degree on the perceived physical mechanism responsible for the instability. These distinctions have not been unambiguous across the various example flows, and the fundamental difference between the two synchronous modes is that one retains the spatio-temporal $Z_{2}$ symmetry and the other breaks it. There is of course a third possibility, that the three-dimensional mode results from a 
complex-conjugate pair of multipliers crossing the unit circle, resulting generally in a quasi-periodic three-dimensional state.

Most wake flow studies are formulated such that there is a single parameter governing the dynamics. As a consequence, for any particular problem, there is only one three-dimensional mode which is first to bifurcate from the two-dimensional state as the parameter is varied. Recently, a different flow with exactly the same symmetry (spatiotemporal $Z_{2} \times O(2)$ ) has been studied, both experimentally and numerically (Vogel, Hirsa \& Lopez 2003; Blackburn \& Lopez 2003b). This flow in a rectangular cavity is driven by the periodic oscillation of one wall while the other cavity walls remain stationary. A vortex 'roller' forms at opposite sides of the cavity with each stroke of the oscillating wall. In this problem there are two (dynamic) parameters, the amplitude and frequency of the wall oscillation, as well as geometric parameters. The ability to vary more than one parameter allows the system to have different primary instability modes of the two-dimensional state in different parameter regimes. In particular, the two synchronous modes and the quasi-periodic mode have each been predicted from Floquet analysis (Blackburn \& Lopez 2003b) to result from primary bifurcations of the two-dimensional state in different regions of parameter space. Further, Marques, Lopez \& Blackburn (2004) considered general systems with spatio-temporal symmetry $Z_{2} \times O(2)$, and concluded that the two synchronous modes and the quasi-periodic mode are the only generic codimension-one bifurcations from two-dimensional to three-dimensional flow. This motivates us to revisit the wake problem and in particular re-examine the role of complex-conjugate Floquet multipliers. Barkley \& Henderson (1996), in their Floquet analysis of the circular cylinder wake identified a mode with complex-conjugate Floquet multipliers, but for the range of Reynolds numbers considered, these had moduli less than one, and so they did not examine it in detail.

\section{Symmetries and the half-period-flip map}

Formally, the spatio-temporal symmetry, $H$, of the two-dimensional flow illustrated in figure 1 operates on the velocity $\boldsymbol{U}(\boldsymbol{x}, t)=(U, V, W)(x, y, z, t)$ as

$$
H \boldsymbol{U}(\boldsymbol{x}, t)=K_{y} \boldsymbol{U}(\boldsymbol{x}, t+T / 2)=(U,-V, W)(x,-y, z, t+T / 2),
$$

and the base flow is $H$-symmetric: $H \boldsymbol{U}(\boldsymbol{x}, t)=\boldsymbol{U}(\boldsymbol{x}, t) . K_{y}$ is a spatial reflection: $y \rightarrow-y$, $V \rightarrow-V$, and $T$ is the fundamental period of the flow. This period is imposed in nonautonomous cases, such as the periodically driven cavity (Vogel et al. 2003; Blackburn \& Lopez 2003b), where the forcing provides two parameters. In autonomous cases, such as bluff body wakes, the period is dynamically determined; it is a function of Reynolds number, $R e=U_{\infty} D / \nu$, where $U_{\infty}$ is the freestream speed and $D$ is the cylinder diameter. The action of $H$ on the vorticity, $\boldsymbol{\Omega}=\left(\Omega_{x}, \Omega_{y}, \Omega_{z}\right)$, is

$$
H \boldsymbol{\Omega}(\boldsymbol{x}, t)=K_{y} \boldsymbol{\Omega}(\boldsymbol{x}, t+T / 2)=\left(-\Omega_{x}, \Omega_{y},-\Omega_{z}\right)(x,-y, z, t+T / 2) .
$$

Although the base flows are both two-dimensional and two-component, and hence $\partial_{z}=0$ and $W=0, W$ is included in (2.1) because we will be studying symmetry properties of three-dimensional instabilities.

A standard tool for the analysis of $T$-periodic flows is the Poincaré map. The stability of perturbations to a limit cycle may be examined by determining their behaviour at successive periods $n T, n \in \mathbb{N}$, thus exchanging the stability analysis of a limit cycle in a continuous dynamical system (ODE or PDE) for the simpler problem of the stability analysis of a fixed point of a map. Let $\phi\left(t ; x_{0}, t_{0}\right)$ be the solution of the continuous system, 


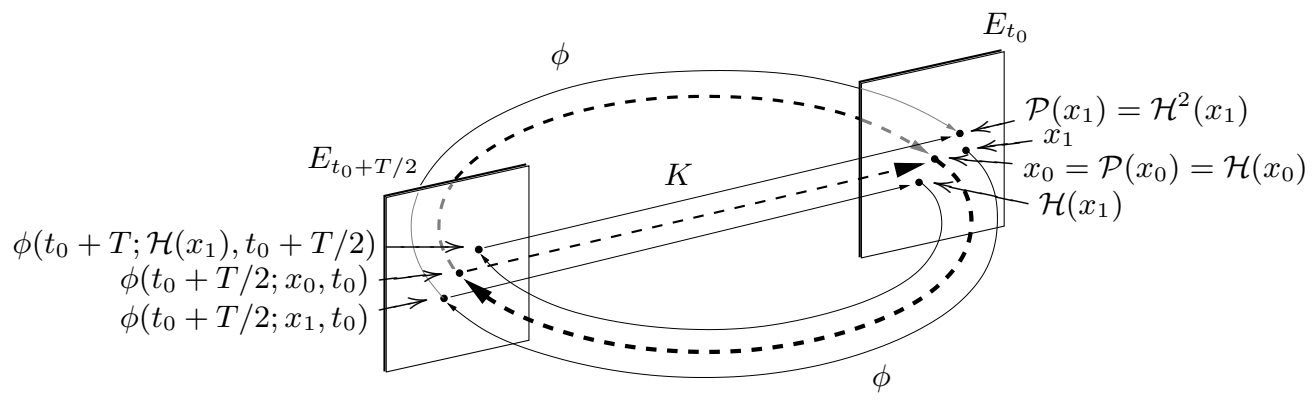

Figure 2. The $T$-periodic Poincaré return map $\mathcal{P}$ and the half-period-flip map $\mathcal{H} . E_{t_{0}}$ is the Poincaré section of the flow for an arbitrary starting time $t_{0} ; E_{t_{0}+T / 2}$ is another section, displaced in time by the half-period $T / 2 . K$ is a spatial symmetry. The basic state (on dashed-line limit cycle) is $x_{0}$, a fixed point of $\mathcal{P}$ and $\mathcal{H} ; x_{1}$ is an arbitrary initial point, close to $x_{0} ; x_{1}, \mathcal{P}\left(x_{1}\right)$ and $\mathcal{H}\left(x_{1}\right)$ are all different, although $\mathcal{P}\left(x_{1}\right)=\mathcal{H}^{2}\left(x_{1}\right)$.

evolving from a set of initial conditions $\left(x_{0}, t_{0}\right)$. The Poincaré map of $x_{0}$ is

$$
x_{0} \mapsto \mathcal{P}\left(x_{0}\right)=\phi\left(t_{0}+T ; x_{0}, t_{0}\right) .
$$

If a periodic solution (a fixed point of the Poincaré map) exists, we can linearise around it to obtain a linear operator $L_{\mathcal{P}}$ (the linearised Poincaré map) and compute its eigenvalues, $\mu_{\mathcal{P}}$, and eigenfunctions. The eigenvalues are the Floquet multipliers of linear perturbations to the periodic solution, while the eigenfunctions are the corresponding time-periodic eigenfunctions evaluated at starting phase $t_{0}$.

A periodic solution loses stability when at least one Floquet multiplier crosses the unit circle; in the absence of symmetries, this may happen in three different ways: $(i) \mu_{\mathcal{P}} \rightarrow$ +1 , saddle-node bifurcation (synchronous); (ii) $\mu_{\mathcal{P}} \rightarrow \mathrm{e}^{ \pm \mathrm{i} \theta}$, Neimark-Sacker bifurcation (quasi-periodic); (iii) $\mu_{\mathcal{P}} \rightarrow-1$, period-doubling bifurcation.

Symmetries dramatically change this picture; given a symmetry group $\mathcal{G}$, the possible bifurcation scenarios are found by analysing the joint representations of $L_{\mathcal{P}}$ and $\mathcal{G}$. The analysis of the influence of purely spatial symmetries in bifurcations and in the corresponding normal forms is well established. For example, let us consider how the bifurcation of a single eigenvalue $\mu_{\mathcal{P}}=+1$ is affected by symmetries. In the presence of a spatial $Z_{2}$ symmetry, the saddle-node bifurcation becomes a pitchfork. And if the symmetry group is $O(2)$, we obtain a pitchfork of revolution (Golubitsky et al. 1988; Iooss \& Adelmeyer 1998; Chossat \& Lauterbach 2000).

The corresponding analysis for spatio-temporal symmetries is more recent. For a $Z_{2}$ spatio-temporal symmetry, as in the problems we are analysing, instead of the Poincaré map, one can alternatively use the half-period-flip map

$$
x_{0} \mapsto \mathcal{H}\left(x_{0}\right)=K \phi\left(t_{0}+T / 2 ; x_{0}, t_{0}\right),
$$

which is simply the action of the space-time symmetry (2.1) on an arbitrary solution and $K$ is a spatial reflection. This technique goes back to Swift \& Wiesenfeld (1984). For this and more complicated spatio-temporal symmetries, the theory is nontrivial, and has been recently, and extensively, developed (e.g. Rucklidge \& Silber 1998; Lamb \& Melbourne 1999; Lamb, Melbourne \& Wulff 2003, and references therein).

The Poincaré map and the half-period-flip map are illustrated diagrammatically in figure 2. A fundamental point, exploited in the present work (and in the aforementioned works on spatio-temporal symmetries), is that since $K^{2}=I$, it follows that $\mathcal{P}=\mathcal{H}^{2}$. The 
Floquet multipliers $\mu_{\mathcal{P}}$ for $\mathcal{P}$ are the squares of those for $\mathcal{H}$, i.e. $\mu_{\mathcal{P}}=\mu_{\mathcal{H}}^{2}$. The stability analysis and the normal forms can be computed for $\mathcal{H}$, and the corresponding results for $\mathcal{P}$ can easily be obtained using $\mathcal{P}=\mathcal{H}^{2}$. This process has two important advantages: first, the space-time symmetry $H$ is the half-period-flip map, so it is naturally included in the analysis; second, for the numerical stability analysis, the time integration for $\mathcal{H}$ is only computed over half the forcing period, so the computational cost is halved.

Lamb \& Melbourne (1999) and Wulff, Lamb \& Melbourne (2001) have identified many of the bifurcations that a dynamical system with spatio-temporal symmetries can undergo. Marques et al. (2004) have computed the corresponding normal forms for centre manifolds of dimension 2 and 4, including all the codimension-one bifurcations, that a dynamical system with spatial symmetry $O(2)$ and spatio-temporal symmetry $Z_{2}$ can undergo. Regardless of the physical instability mechanisms involved, for the transition from two-dimensional to three-dimensional flow only three different codimension-one bifurcations are possible. Two of them are synchronous $\left(\mathcal{F}_{2}^{+}\right.$and $\left.\mathcal{F}_{2}^{-}\right)$, corresponding to real eigenvalues +1 and -1 of $\mathcal{H}$ and two-dimensional centre manifolds. The third is a quasi-periodic bifurcation $\left(\mathcal{F}_{4}^{c}\right)$ with complex-conjugate eigenvalues and a four-dimensional centre manifold.

Codimension-one bifurcations are particularly important because they are the bifurcations generically obtained when a single parameter is varied. In order to obtain codimension-two bifurcations, at least two independent parameters must be simultaneously tuned. Although codimension-two (or higher) bifurcations are more difficult to obtain, they display much richer dynamics, and often act as organising centres for the dynamics in large regions of parameter space (e.g. see Mullin 1993; Marques, Lopez \& Shen 2002; Marques, Gelfgat \& Lopez 2003; Lopez \& Marques 2003). The primary bifurcations are generically governed by codimension-one bifurcations, but the dynamics following the primary bifurcation (e.g. secondary and global bifurcations) are often organised by nearby codimension-two or higher bifurcations. In the present paper we focus on the occurrence of the three codimension-one bifurcations $\mathcal{F}_{2}^{+}, \mathcal{F}_{2}^{-}$and $\mathcal{F}_{4}^{c}$ in different wake flows. The details of the joint representations of the spatio-temporal $Z_{2}$ and spatial $O(2)$ symmetries, the centre manifold reductions and the associated normal forms are in Marques et al. (2004).

The stability of the periodic two-dimensional base flow is analysed by linearising the half-period-flip map $\mathcal{H}$. As the base flow is two-dimensional, we can Fourier expand the perturbed velocity field $\boldsymbol{u}^{\prime}$ in the $z$ direction and analyse the stability of each Fourier mode independently for different spanwise wavelengths $\lambda$. The form of one of these Fourier modes is $\boldsymbol{u}^{\prime}=\boldsymbol{u}(x, y, t) \mathrm{e}^{\mathrm{i} \beta z}$, where $\beta=2 \pi / \lambda$. When the flow is unstable to three-dimensional perturbations $(\beta \neq 0)$, the real and imaginary parts of $\boldsymbol{u}^{\prime}$ are linearly independent, and their linear combinations form a two-dimensional linear space. Therefore, the dimension of the centre manifold is even. In the two synchronous bifurcation cases, the centre manifold is two-dimensional, and in the quasi-periodic case it is four-dimensional.

\section{Bluff body wakes}

\subsection{Circular cylinder wakes}

The long and short wavelength instabilities of the two-dimensional wakes of the circular cylinder (Williamson 1988, 1996; Barkley \& Henderson 1996) are synchronous with $\mu_{\mathcal{P}}$ real and positive. Respectively, these long and short wavelength modes are known as modes A and B. Figure 3 shows visualisations of modes A and B for the cylinder wake, 


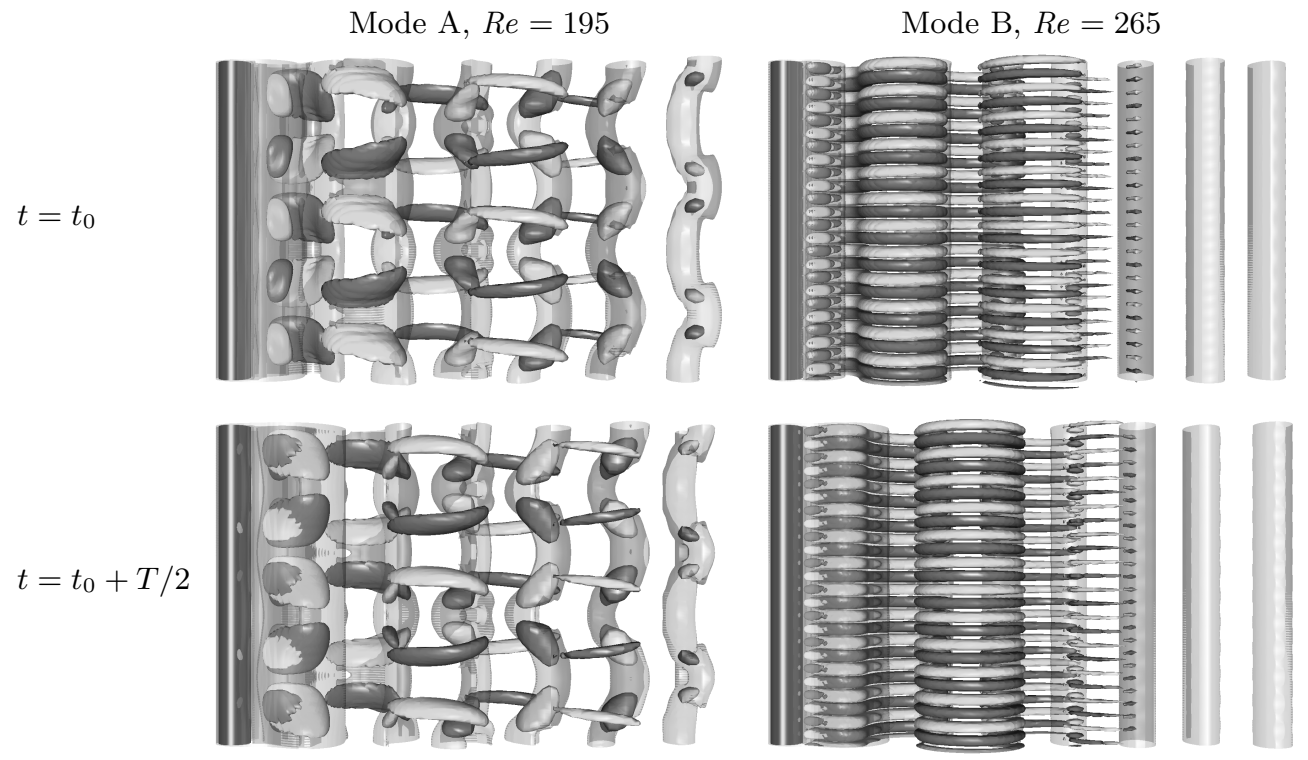

FiguRE 3. Vorticity isosurfaces for the synchronous wake modes of the circular cylinder, shown for a $10 D$ spanwise domain extent, and viewed from the cross-flow direction. Translucent isosurfaces are for spanwise vorticity component, solid surfaces are for streamwise component.

obtained through direct numerical simulation with restricted spanwise periodic length, at Reynolds numbers slightly above onset for the two modes. The non-translucent isosurfaces in the figure are of the (streamwise) $x$ component of vorticity. For a $H$-symmetric flow, from (2.2), the $x$-vorticity changes sign with $t \rightarrow t+T / 2$ and $y \rightarrow-y$ at any fixed $(x, z)$. The figure, with views in the (cross-flow) $y$ direction, shows this to be the case for mode $\mathrm{A}$, whereas for mode $\mathrm{B}$, the sign of $x$-vorticity does not change with $t \rightarrow t+T / 2$ and $y \rightarrow-y$, i.e. mode $\mathrm{A}$ is $H$-invariant and mode $\mathrm{B}$ is not.

Previous Floquet analyses have been based on the Poincaré map, and for both of these synchronous modes the multipliers cross the unit circle at $\mu_{\mathcal{P}}=+1$. Floquet analysis based on the half-period-flip map (see Appendix A for details) shows that the $H$-invariant mode A bifurcates at $\mu_{\mathcal{H}}=+1$, while mode B has $\mu_{\mathcal{H}}=-1$ (giving $\left.\mu_{\mathcal{P}}=\mu_{\mathcal{H}}^{2}=+1\right)$. Note that $\mu_{\mathcal{H}}=-1$ is not a period doubling bifurcation for $\mathcal{P}$ (although it is a period doubling bifurcation for the map $\mathcal{H}$, whose period is half the period of $\mathcal{P}$ ): it is an $H$-symmetry breaking bifurcation.

The synchronous bifurcations where modes A and B originate are pitchforks of revolution, because they break the spanwise translation invariance $(S O(2)$ symmetry), leading to a continuous family of solutions that are parametrised by their phase in $z$. Furthermore, these solutions preserve the spanwise reflection $K_{z}$ at appropriate discrete points.

The Floquet multipliers for the two-dimensional wake of a circular cylinder, computed at $R e=280$, show that the two-dimensional basic state is unstable to both modes $\mathrm{A}$ and $\mathrm{B}$, while there is an intermediate-wavenumber mode (or modes) with complex-conjugate pair Floquet multipliers to which the basic state is stable, i.e. $|\mu|<1$ (Barkley \& Henderson 1996). We have computed and plotted in figure $4(a)$ the absolute values of the Floquet multipliers for the linearised Poincaré map, $\left|\mu_{\mathcal{P}}\right|$, as functions of wavenumber $\beta$ at this Re (solid circles) and compared these with the results from Barkley \& Henderson (1996) (open squares), and the two agree. The onset of mode A is a primary bifurcation of the periodically shedding two-dimensional wake, occurring at $R e \approx 188$, and mode $\mathrm{B}$ is a 
(a)

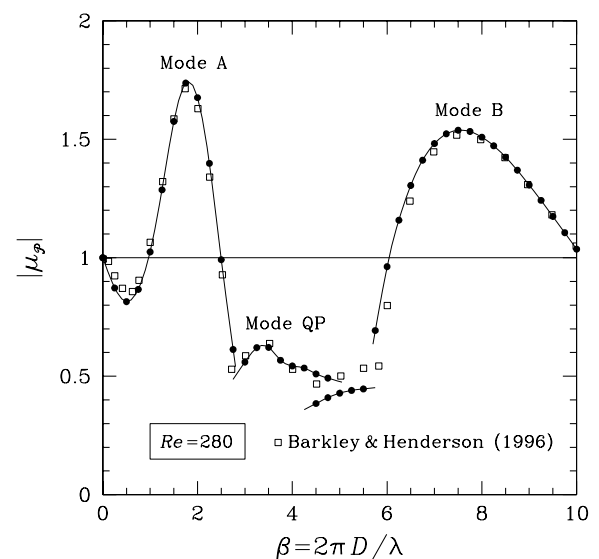

(b)

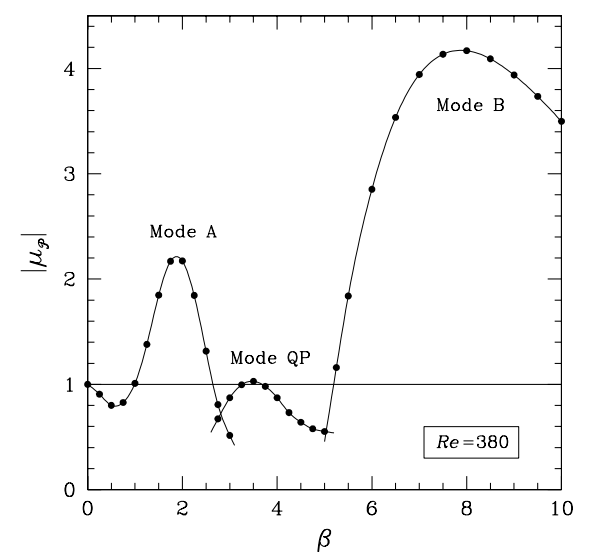

Figure 4. Moduli of the Floquet multipliers, $\left|\mu_{\mathcal{P}}\right|$, for the three-dimensional instability modes of the two-dimensional wake of a circular cylinder at $(a) R e=280$ and $(b) R e=380$. The $R e=280$ results are compared with those of Barkley \& Henderson (1996) at the same Re (open squares). Multipliers for mode QP occur in complex-conjugate pairs.

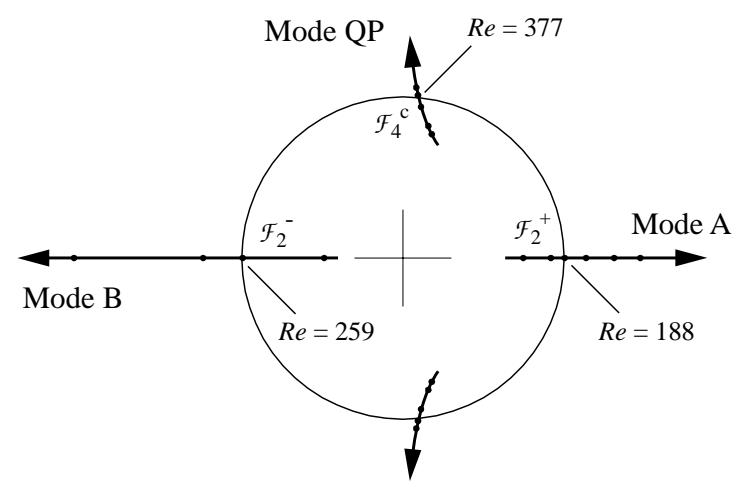

FiguRE 5. Loci with increasing $R e$ of the Floquet multipliers of the linearised $\mathcal{H}$-map of the three codimension-one bifurcations of the two-dimensional $T$-periodic circular cylinder wake, plotted over the unit circle in the complex plane.

secondary bifurcation at $R e \approx 259$, so that in practice it is not observed as a pure mode. Figure $4(b)$ shows $\left|\mu_{\mathcal{P}}\right|$ for $R e=380$, at which value the quasi-periodic mode, $\mathrm{QP}$, has just become critical. At $R e=380$ the growth rate (modulus of the Floquet multiplier) for mode $\mathrm{B}$, the secondary mode, is about twice that of the primary mode A, reversing the ranking at $R e=280$. The quasi-periodic modes appear at tertiary bifurcations from the $T$-periodic two-dimensional basic state at $R e \approx 377$.

How the Floquet multipliers for the linearised $\mathcal{H}$ map, $\mu_{\mathcal{H}}$, cross the unit circle as $R e$ is varied is shown in figure 5 . Using the $\mathcal{H}$ map, it is clear that mode B is a symmetrybreaking mode and that the quasi-periodic mode is close to being a period-doubling bifurcation, with critical complex-conjugate multipliers $\mu_{\mathcal{H}}=\mathrm{e}^{ \pm \mathrm{i} 0.470 \pi}$, of multiplicity two since the spanwise $O(2)$ symmetry is broken. A period-doubling bifurcation would occur through a codimension-two bifurcation corresponding to a 1:4 resonant $\mathcal{F}_{4}^{c}$ bifurcation, with $\mu_{\mathcal{H}}=\mathrm{e}^{ \pm \mathrm{i} 0.5 \pi}$ corresponding to $\mu_{\mathcal{P}}=-1$ with multiplicity four (Marques et al. 2004). The theory for the suppression of period doubling with a simple Floquet 


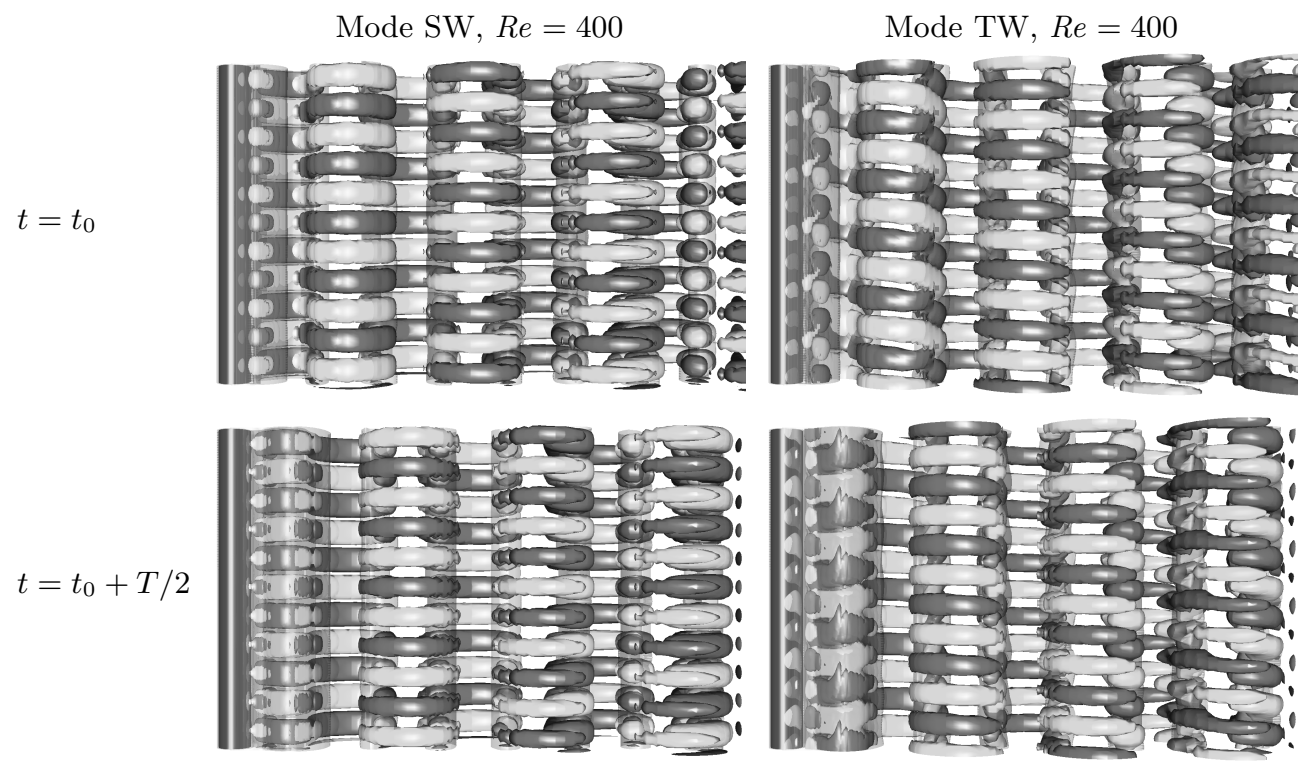

FiguRE 6. Vorticity isosurfaces for the quasi-periodic wake modes of the circular cylinder, shown for a $10 D$ spanwise domain extent, and viewed from the cross-flow direction. Translucent isosurfaces are for spanwise vorticity component, solid surfaces are for streamwise component.

multiplier at $\mu_{\mathcal{P}}=-1$ (Swift \& Wiesenfeld 1984) does not apply. So, while period doubling is possible, it is not generic in a single parameter system and it appears that no true subharmonic mode has been reported for these wake flows.

With only a single parameter to vary, $R e$, only one of the three possible codimensionone bifurcations will be observed as a primary bifurcation, in this case mode A. For other flows, or by varying a second parameter (which could be geometric), either modes QP or $\mathrm{B}$ might become primary in some region of parameter space. In the periodically driven cavity, with the forcing amplitude and frequency as two available parameters, there are regimes where each of the three codimension-one bifurcations are primary (Blackburn \& Lopez 2003b). Nevertheless, secondary and tertiary bifurcations from an unstable state can have a profound influence on the flow dynamics. For example, in the vortex breakdown problem in a cylindrical container the tertiary bifurcated modes from the base state eventually become dominant as $R e$ is increased; this has been observed experimentally (Stevens, Lopez \& Cantwell 1999) and analysed numerically (Lopez, Marques \& Sanchez 2001; Blackburn \& Lopez 2002; Blackburn 2002).

At the quasi-periodic bifurcation, two distinct solution branches emerge simultaneously, one corresponding to (modulated) travelling waves, TW, and the other to (modulated) standing waves, SW; both are modulated by the time-periodic base state. Figure 6 shows visualisations of the quasi-periodic modes SW and TW for the cylinder wake, obtained through direct numerical simulation at $R e=400$, slightly above onset for the quasi-periodic modes $(R e=377)$. Again, as for figure 3, the non-translucent isosurfaces in the figure are of the (streamwise) $x$-component of vorticity. The TW have (slightly) oblique alternating streamwise vortices, and those on opposite sides of the wake are interlaced in the spanwise direction, a distinguishing feature of this mode. The TW propagating in the $+z$ direction is shown; the $-z$-propagating TW is obtained by applying the reflection $K_{z}$ to the $+z$-TW. After one period $T$ the flow is identical, but translated in the spanwise direction. After half a period $T / 2$, we recover the same flow after a re- 


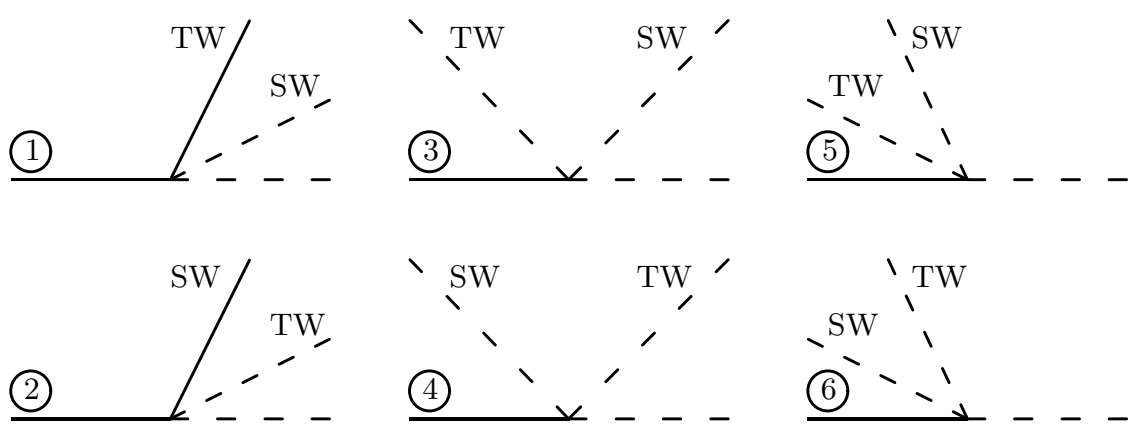

FIGURE 7. Bifurcation diagrams corresponding to the six scenarios in the QP bifurcation. Solid (dashed) lines represent stable (unstable) states, the horizontal line corresponds to the $T$-periodic base state. The horizontal axis is the bifurcation parameter $(R e)$, and the vertical axis is the amplitude squared of the $3 \mathrm{D}$ components of the solution.

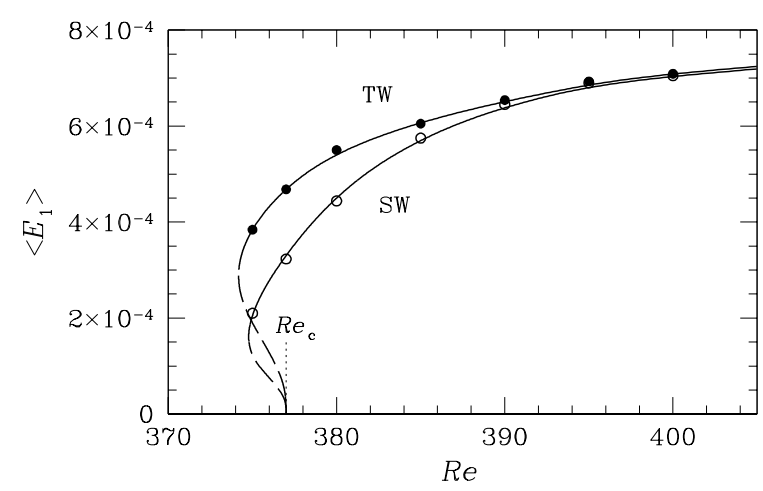

Figure 8. Time-average kinetic energies in the first spanwise Fourier mode of the TW and SW nonlinear solutions of the wake of a circular cylinder, as functions of Reynolds number. Solid (open) circles correspond to stable (unstable) solutions, relative to each other. Dashed lines indicate the unstable segments of the two solution branches, taken individually.

flection $K_{y}$ and an appropriate translation in the spanwise direction. The standing wave $\mathrm{SW}$ does not have oblique streamwise vortices, and is $K_{z}$ invariant.

Let us compare these results with the normal form analysis for the codimension-one bifurcation with complex eigenvalues, $\mathcal{F}_{4}^{c}$, for systems with spatial symmetry $O(2)$ and spatio-temporal symmetry $Z_{2}$ (Marques et al. 2004). In the $\mathcal{F}_{4}^{c}$ bifurcation, there is a pair of complex-conjugate Floquet multipliers, $\mu_{\mathcal{H}}=\mathrm{e}^{ \pm \mathrm{i} \theta / 2}, \theta \in(0,2 \pi)$, of multiplicity two (i.e. a total of four). Depending on the particulars of the problem, there are six possible scenarios. The associated bifurcation diagrams are shown in figure 7 . At the bifurcation point three new solutions appear simultaneously: a pair of modulated travelling waves, moving in the positive and negative $z$ direction (and plotted as the same line TW), and a modulated standing wave SW. The bifurcation diagrams shown in figure 7 are in terms of the bifurcation parameter Re (horizontal axis) and the amplitude squared (energy) of the three-dimensional components of the solutions considered.

The six scenarios differ in the stability properties and subcritical/supercritical character of the TW and SW solutions. Scenarios 5 and 6 seem identical, but differ in the number of unstable eigenvalues for TW and SW; the solution with larger slope has a single unstable eigenvalue, and the other has two. For the cylinder wake, the bifurcation to the QP modes is subcritical, as shown in figure 8, where the variations with $R e$ 


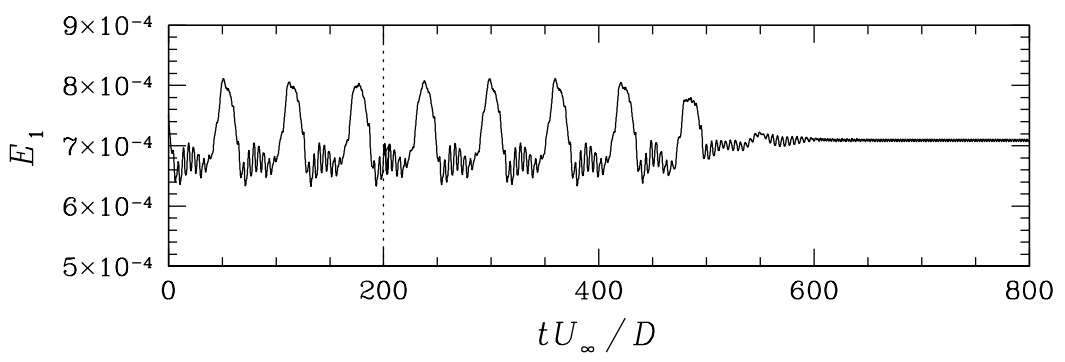

Figure 9. Time series of kinetic energy in the first spanwise Fourier mode for a quasi-periodic circular cylinder wake. Initially, the flow is in a SW state, in a subspace with spanwise reflection symmetry. At $t U_{\infty} / D=200$, it is perturbed with a small amount of white noise, after which it evolves to a stable, asymmetric, TW state.

of the time-average of the kinetic energies in the first spanwise Fourier mode, $\left\langle E_{1}\right\rangle$, of the TW and SW solutions are plotted. Both TW and SW are subcritical and unstable, corresponding to either scenarios 5 or 6 in figure 7. Floquet analysis cannot distinguish between scenarios 5 or 6 and nonlinear information is required. Even though modes A and $\mathrm{B}$ have already bifurcated from the basic state at the Re values where the quasi-periodic modes also bifurcate, we can still make nonlinear computations in which modes A and B are not present by restricting the wavelengths used in the computations. This is possible in this problem because of the well-separated spectra associated with the various modes (see figure 4). Initiating nonlinear computations with a symmetric combination of the critical eigenvectors at $R e$ near the critical value for the quasi-periodic bifurcation (see the Appendix for details), one can compute in an SW-invariant subspace. Perturbing a nonlinear SW state, we can determine whether SW is stable relative to TW. We have done this over the range of Re shown in figure 8, and in all cases SW evolves towards TW. An example of such an evolution is provided in figure 9. The nonlinear information that TW is stable relative to SW determines that scenario 6 in figure 7 is the appropriate bifurcation diagram for the quasi-periodic bifurcation of the circular cylinder.

For TW, both advancing in time by the period $T$ and the action of the spatio-temporal symmetry $H$ are equivalent to $z$-translations. Although $H$ symmetry is not preserved, there is still a preserved spatio-temporal symmetry, corresponding to $H$ in a frame of reference translating in $z$ at the phase speed. Each SW is a linear combination of two equal amplitude modulated travelling waves, travelling in opposite directions. They possess a $K_{z}$ symmetry. Translation invariance in the spanwise direction and $H$ symmetry are broken, and SW do not retain any spatio-temporal symmetry. The numerically computed solutions in the wake of the circular cylinder shown in figure 6 exhibit all these various symmetries, and agree with the mathematical model $\mathcal{F}_{4}^{c}$.

\subsection{Other wake flows}

Studies of wakes of symmetric bluff bodies are usually limited in the sense that they only allow for a single control parameter, Re. As noted in $\S 3.1$, only one of the three possible codimension-one bifurcations (i.e. corresponding to $\mathcal{F}_{2}^{+}, \mathcal{F}_{2}^{-}$or $\mathcal{F}_{4}^{c}$ ) will be primary for a given bluff body, although one of the remaining two could instead be made primary if another parameter, perhaps geometric, is introduced into the problem. For example, the circular cylinder could be thought of as one of a family of ellipses, with the ratio of minor to major axes being the second parameter. Wake flows of symmetrical bodies other than the circular cylinder have been studied, e.g. the square cylinder wake (Robichaux et al. 1999) and the flat plate wake (Meiburg \& Lasheras 1988; Lasheras \& Meiburg 


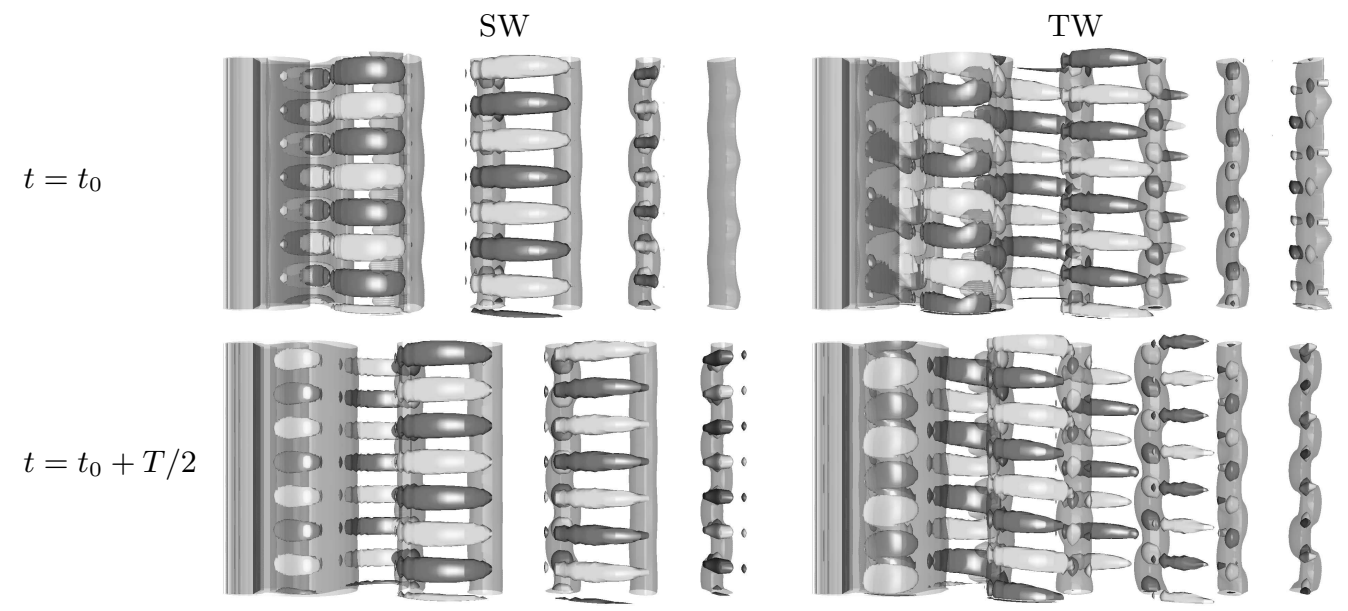

FIGURE 10. Vorticity isosurfaces for the quasi-periodic wake modes of the square cylinder at $R e=220$, shown for a $10 D$ spanwise domain extent, and viewed from the cross-flow direction. Translucent isosurfaces are for spanwise vorticity component, solid surfaces are for streamwise component.

1990; Julien et al. 2003). In both of these cases, there are, as in the circular cylinder case, two distinct synchronous three-dimensional modes, one that preserves $H$-symmetry and one that breaks it. The symmetric mode 2 of the flat plate has the same symmetry as mode $\mathrm{A}$ of the cylinders and mode $\mathrm{B}$ of the periodically driven cavity, whereas the antisymmetric mode 1 of the flat plate has the same symmetry as mode B of the cylinders and mode A of the cavity. While symmetry properties of these two modes with long and short wavelengths coincide for the square and circular cylinders, for the flat plate the two modes have comparable wavelengths.

The square cylinder wake also has intermediate-wavelength modes with complexconjugate pair multipliers, much like the circular cylinder wake. Originally, Robichaux et al. (1999) interpreted these intermediate-wavelength modes as subharmonic (perioddoubled), but this was probably related to their use of a power method with a single vector to perform the numerical Floquet analysis; using only a single vector for a bifurcation problem with a multi-dimensional centre manifold can lead to erroneous conclusions. The analysis was revisited in Blackburn \& Lopez (2003a), where it was demonstrated that these wake modes are in fact quasi-periodic.

Figure 10 shows nonlinear TW and SW wake solutions for the square cylinder wake; as for the circular cylinder case, these were computed by restricting the spanwise extent of the computational domain to the wavelength of the quasi-periodic modes - otherwise, the synchronous modes, which become unstable at lower Reynolds number, would have dominated. It is apparent that the symmetries of these modes are identical to those corresponding to TW and SW of the circular cylinder. In contrast however, the quasiperiodic bifurcations for the square cylinder are both supercritical; this is seen in the time-averaged kinetic energy $\left\langle E_{1}\right\rangle$, plotted in figure 11, where we find that TW has higher energy and so is stable relative to SW (we have also determined relative stability by perturbing the solutions, as was done for the circular cylinder). So, the bifurcation diagram for the quasi-periodic bifurcations of the square cylinder is scenario 1 in figure 7 .

In any real experiment with the wake flows we have discussed (circular and square cylinder, and flat plate), the $O(2)$ symmetry is only approximate, due to the presence of spanwise endwalls at finite distance, which breaks the translation invariance $S O(2)$, leav- 


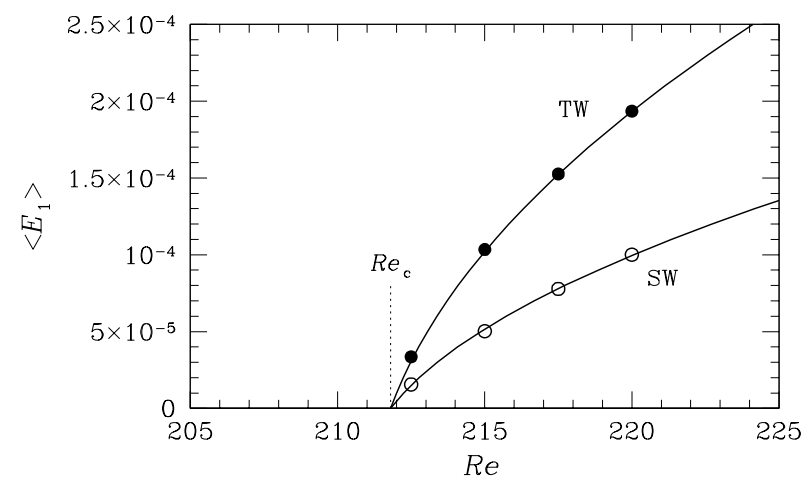

FiguRE 11. Time-average kinetic energies in the first spanwise Fourier mode of the TW and SW instability modes of the wake of a square cylinder, as functions of Reynolds number. The energy of the SW is smaller, and in accordance with the theory, the SW flow is unstable to perturbations.

ing only invariance to reflections about the mid-span, $Z_{2}$. Nevertheless, the experimental observations show a remarkable agreement with the $O(2)$ symmetric theory when the cylinder diameter to length ratio is large. Sheard, Thompson \& Hourigan $(2003,2004)$ have considered bluff ring wakes, with $O(2)$ symmetry corresponding to rotations and reflections in the plane of the ring. In periodic shedding, this wake lacks the space-time $Z_{2}$ symmetry of the cylinder wakes. For large aspect ratio (ratio of major to minor diameters of the ring), Sheard et al. $(2003,2004)$ have found two synchronous modes that have the same characteristics as modes A and B of cylinder wakes. They also report a third mode, mode $\mathrm{C}$, which they describe as being subharmonic, with characteristics similar to the subharmonic mode in Robichaux et al. (1999). However, in the limit of large aspect ratio, ring curvature goes to zero, the ring asymptotes to a circular cylinder, the space-time $Z_{2}$ symmetry of the cylinder wake is recovered, and period-doubling bifurcations become codimension-two. Since both sets of workers used essentially the same single-vector power method in their numerical analysis, it would be interesting to re-examine these recent results for the ring, particularly those at high aspect ratio, with a numerical technique appropriate to dealing with a multi-dimensional centre manifold. The point is that since the problem (for any ring aspect ratio) has exact $O(2)$ symmetry, the centre manifold for any bifurcation breaking $O(2)$, i.e. leading to three-dimensional flow, must be of even dimension, and so cannot be spanned by a single vector. In general, the one-dimensional power method technique is only appropriate if one knows in advance, or can determine through other information, that the Floquet multipliers are real.

\subsection{Physical mechanisms and mode symmetries}

Thus far, we have not addressed the issue of physical mechanisms for the various modes and bifurcations. Two reasons for this are that $(i)$ our analysis, which is general, does not need to account for physical mechanisms, only symmetries, and (ii) while the physical characteristics of the various modes (e.g. the long-wavelength synchronous modes) appear to have some similarities across the various flows considered, the actual physical mechanisms involved remain a topic of some controversy. $\dagger$ However, there is no correlation between spanwise wavelength of three-dimensional instabilities and the associated

$\dagger$ For the circular cylinder wake and the driven cavity, candidate physical mechanisms for the synchronous modes are discussed at length by Blackburn \& Lopez (2003b). For the flat plate wake, an extensive discussion is presented by Julien et al. (2003). 
symmetries: in the driven cavity, the long-wavelength instability occurs through the $\mathcal{F}_{2}^{-}$ bifurcation, while for the circular and square cylinder wakes it is associated with $\mathcal{F}_{2}^{+}$. The symmetries, wavelengths, and in general, physical mechanisms, are independent of one another. The unifying property for all two-dimensional symmetric time-periodic flows is their symmetry, which in turn dictates their normal forms and how they can bifurcate to three-dimensional flows - which generically (i.e. when only a single parameter is varied) will be via $\mathcal{F}_{2}^{+}, \mathcal{F}_{2}^{-}$or $\mathcal{F}_{4}^{c}$ bifurcations.

\section{Conclusions}

We have presented a unified description of the bifurcations to three-dimensionality which can occur from a two-dimensional time-periodic base state with space-time reflection symmetry, and the symmetries of the resulting bifurcated states. The analysis shows that there are exactly three codimension-one bifurcations from two-dimensional to threedimensional flow (i.e. bifurcations that are generally observable with variations in a single parameter). Two of these are synchronous, one breaking and the other preserving the space-time symmetry, while the third is quasi-periodic with a bifurcated state that may be manifest as either a modulated travelling wave or a modulated standing wave. These three bifurcations have been observed in the experimental and computational studies of autonomous bluff body wakes and the non-autonomous periodically driven cavity flow. Furthermore, from the analysis of these different flows, it is apparent that the physical characteristics of the flows (e.g. spanwise wavelength, roller core deformations, structure of streamwise braids, etc.) are not correlated with the type of bifurcation responsible for the three-dimensional flows (synchronous or quasi-periodic) nor with their symmetries (e.g. preserving or breaking the space-time symmetry). This is to be expected, as the characteristics of the base flow (two-dimensional time-periodic with space-time reflection) are generic properties of flows, independent of the flows' physical characteristics, in the same way that the simpler Hopf and pitchfork bifurcations are common to a vast array of flows and come about by different physical flow instabilities.

The use of the half-period-flip map in the bifurcation analysis, and in particular for its novel application in the Floquet stability analysis reported here, allows unambiguous prediction of the essential characteristics of the bifurcated states, and this comes with a considerable computational savings over conventional analysis based on the Poincaré map. Further, any complete description of the bifurcation problem requires a basis that spans the corresponding centre manifold.

This work was supported by the Australian Partnership for Advanced Computing's Merit Allocation Scheme, the Australian Academy of Science's International Scientific Collaborations Program, MCYT grant BFM2001-2350 (Spain), and NSF Grant CTS9908599 (USA).

\section{Appendix A. Floquet analysis with the half-period-flip map}

The theory underlying Floquet analysis as applied to the unsteady Navier-Stokes equations has been previously presented in detail for problems with $O(2)$ spatial symmetry (e.g. Barkley \& Henderson 1996; Robichaux et al. 1999; Blackburn \& Lopez 2003b). Floquet stability analysis studies the evolution of a three-dimensional perturbation $\boldsymbol{u}^{\prime}$ to a $T$-periodic 'base flow' $\boldsymbol{U}: \boldsymbol{u}=\boldsymbol{U}+\boldsymbol{u}^{\prime}$. The linearised equivalent of the incompressible Navier-Stokes equations for an infinitesimal perturbation $\boldsymbol{u}^{\prime}$ can be written as

$$
\partial_{t} \boldsymbol{u}^{\prime}=\left(\partial_{\boldsymbol{U}} \boldsymbol{N}+\boldsymbol{L}\right) \boldsymbol{u}^{\prime},
$$


where $\partial_{\boldsymbol{U}} \boldsymbol{N}+\boldsymbol{L}$ represents the linearisation (Jacobian) of $\boldsymbol{N}+\boldsymbol{L}$ (the nonlinear operator $\boldsymbol{N}$ contains contributions from both advection and pressure terms, while the linear operator $\boldsymbol{L}$ corresponds to viscous diffusion) about the base flow $\boldsymbol{U} ; \partial_{\boldsymbol{U}} \boldsymbol{N}$ is the $T$-periodic linear operator obtained from $\boldsymbol{N}$ by replacing nonlinear advection terms with their linearised equivalent $\boldsymbol{u}^{\prime} \cdot \boldsymbol{\nabla} \boldsymbol{U}+\boldsymbol{U} \cdot \boldsymbol{\nabla} \boldsymbol{u}^{\prime}$. The operator $\partial_{\boldsymbol{U}} \boldsymbol{N}+\boldsymbol{L}$ is equivalent to the flow $\phi$ in $(2.3),(2.4)$, thus

$$
\begin{aligned}
& \boldsymbol{u}_{0}^{\prime} \mapsto \mathcal{P}\left(\boldsymbol{u}_{0}^{\prime}\right)=\phi\left(t_{0}+T ; \boldsymbol{u}_{0}^{\prime}, t_{0}\right), \\
& \boldsymbol{u}_{0}^{\prime} \mapsto \mathcal{H}\left(\boldsymbol{u}_{0}^{\prime}\right)=K \phi\left(t_{0}+T / 2 ; \boldsymbol{u}_{0}^{\prime}, t_{0}\right),
\end{aligned}
$$

where now $\mathcal{P}$ and $\mathcal{H}$ represent the linearised Poincaré and half-period-flip maps of the perturbation velocity, respectively. Both $\boldsymbol{U}$ and $\phi$ have the same spatio-temporal symmetries, and $\phi$ is said to be equivariant with respect to $H$-symmetry (2.1) (Golubitsky \& Stewart 2002).

Perturbation solutions $\boldsymbol{u}^{\prime}$ can be written as a sum of Floquet modes, $\check{\boldsymbol{u}}\left(t-t_{0}\right)=$ $\tilde{\boldsymbol{u}}\left(t_{0}\right) \mathrm{e}^{\gamma\left(t-t_{0}\right)}$, where $\tilde{\boldsymbol{u}}\left(t_{0}\right)$ are the $T$-periodic Floquet eigenfunctions of $\phi$ and the constants $\gamma=\sigma+\mathrm{i} \omega$ are Floquet exponents. The Floquet multipliers, which define the growth of Floquet modes over the period $T$, are related to the Floquet exponents by $\mu_{\mathcal{P}}=\mathrm{e}^{\gamma T}$. Floquet multipliers for the half-period-flip map are $\mu_{\mathcal{H}}=\mathrm{e}^{\gamma T / 2}$, so that $\mu_{\mathcal{P}}=\mu_{\mathcal{H}}^{2}$. The time-periodic basic state becomes linearly unstable when one or more Floquet multipliers leave the unit circle.

A convenient means of finding $\mu_{\mathcal{P}}$ and $\tilde{\boldsymbol{u}}$ is to compute the eigenvalues and eigenfunctions of $\mathcal{P}$ : the eigenvalues are the Floquet multipliers and the eigenfunctions are $\tilde{\boldsymbol{u}}\left(t_{0}\right)$. Likewise, $\mu_{\mathcal{H}}$ are the eigenvalues of $\mathcal{H}$, while the eigenfunctions are the same as those for $\mathcal{P}$. Krylov methods are typically used to compute the discrete eigensystem of $\mathcal{P}$, as described in detail by Tuckerman \& Barkley (2000). The same methods are simply adapted to compute the eigensystem of $\mathcal{H}$ : instead of integrating perturbations over the complete period $T$ on each iteration [i.e. iterating $\mathcal{P}\left(\boldsymbol{u}^{\prime}\right)$ ], they are integrated only over $T / 2$, followed by explicit application of the symmetry $K$, thus iterating $\mathcal{H}\left(\boldsymbol{u}^{\prime}\right)$. A side-benefit is that the computational time required for convergence of the eigensystem is typically halved.

We now address the spatial structure of the three-dimensional Floquet instabilities of the $Z_{2} \times O(2)$ flows in question. As a consequence of the two-dimensional geometry and boundary conditions, and the linearity of (A 1), we can Fourier expand $\boldsymbol{u}^{\prime}$ in the $z$ direction and analyse the stability of each Floquet Fourier mode independently for different spanwise wavelengths $\lambda$. Since the base flow is two-dimensional and two-component, two linearly independent expansion functions for $\boldsymbol{u}^{\prime}$ are

$$
\begin{aligned}
& \left(u^{\prime}, v^{\prime}, w^{\prime}\right)(x, y, z, t)=\left(\hat{u}^{\prime} \cos \beta z, \hat{v}^{\prime} \cos \beta z, \hat{w}^{\prime} \sin \beta z\right)(x, y, t), \\
& \left(u^{\prime}, v^{\prime}, w^{\prime}\right)(x, y, z, t)=\left(\hat{u}^{\prime} \sin \beta z, \hat{v}^{\prime} \sin \beta z, \hat{w}^{\prime} \cos \beta z\right)(x, y, t),
\end{aligned}
$$

where $\beta=2 \pi / \lambda$ is a spanwise wavenumber - these break the spanwise $O(2)$ symmetry for $\beta \neq 0$, and are spatially periodic in $z$.

When the critical eigenvalue is real, the centre manifold is generically two-dimensional, as discussed in $\S 2$, and is generated by linear combinations of (A 4) and (A 5). Physically, different linear combinations correspond to translations in the spanwise direction. Any of these linear combinations can be used as the desired eigenfunction in the Floquet analysis.

When the eigenvalues are complex, the centre manifold is generically four-dimensional, and we have two sets of linear generators of the form (A 4) and (A 5). Both sets are mixed by time evolution and also by reflection in the spanwise direction. In this case one cannot 
in general take either (A 4) or (A 5), or even a specific linear combination of the two, for $\boldsymbol{u}^{\prime}$. To do so corresponds to fixing the phase in $z$ and hence imposes the symmetry of a (modulated) standing wave; modulated by the time-periodicity of the basic state. When the multipliers are complex-conjugate, a general linear combination of (A 4) and (A 5) must be used to allow for (modulated) travelling wave solutions. Nevertheless, it can be interesting in nonlinear computations to fix the phase in $z$, and therefore produce modulated standing wave solutions; this is equivalent to restricting the solutions to a $K_{z}$ invariant subspace, and allows the computation of the modulated standing wave solutions in case they are unstable. It should be noted that since the symmetric expansions (A 4 ) and (A 5) preserve their symmetry under the Navier-Stokes equations, full simulations initialised in a symmetric state will remain symmetric unless perturbed asymmetrically it is not necessary to enforce the restriction.

For all the results discussed here, the underlying spatial discretisation employs spectral elements, and the numerical Floquet analysis is based on an Arnoldi method (Barkley \& Henderson 1996; Tuckerman \& Barkley 2000). We refer the reader interested in further detail to Blackburn \& Lopez (2003a) for the circular and square section cylinder wakes, to Blackburn \& Lopez (2003b) for the rectangular periodically driven cavity and to Blackburn (2002) for application to the cylindrical lid-driven cavity.

\section{REFERENCES}

BArkley, D. \& Henderson, R. D. 1996 Three-dimensional Floquet stability analysis of the wake of a circular cylinder. J. Fluid Mech. 322, 215-241.

Barkley, D., Tuckerman, L. S. \& Golubitsky, M. S. 2000 Bifurcation theory for threedimensional flow in the wake of a circular cylinder. Phys. Rev. E 61, 5247-5252.

Benjamin, T. B. 1978 a Bifurcation phenomena in steady flows of a viscous fluid. I. Theory. Proc. R. Soc. Lond. A 359, 1-26.

Benjamin, T. B. $1978 b$ Bifurcation phenomena in steady flows of a viscous fluid. II. Experiments. Proc. R. Soc. Lond. A 359, 27-43.

Blackburn, H. M. 2002 Three-dimensional instability and state selection in an oscillatory axisymmetric swirling flow. Phys. Fluids 14, 3983-3996.

Blackburn, H. M. \& Lopez, J. M. 2002 Modulated rotating waves in an enclosed swirling flow. J. Fluid Mech. 465, 33-58.

Blackburn, H. M. \& Lopez, J. M. 2003a On three-dimensional quasi-periodic Floquet instablities of two-dimensional bluff body wakes. Phys. Fluids 15, L57-60.

Blackburn, H. M. \& Lopez, J. M. $2003 b$ The onset of three-dimensional standing and modulated travelling waves in a periodically driven cavity flow. J. Fluid Mech. 497, 289-317.

Blohm, C. \& Kuhlmann, H. C. 2002 The two-sided lid-driven cavity: experiments on stationary and time-dependent flows. J. Fluid Mech. 450, 67-95.

Chossat, P. \& Iooss, G. 1994 The Couette-Taylor Problem. Springer.

Chossat, P. \& Lauterbach, R. 2000 Methods in Equivariant Bifurcations and Dynamical Systems. World Scientific.

Crawford, J. D. \& KNobloch, E. 1991 Symmetry and symmetry-breaking bifurcations in fluid dynamics. Ann. Rev. Fluid Mech. 23, 341-387.

Cross, M. C. \& Hohenberg, P. C. 1993 Pattern formation outside of equilibrium. Rev. Mod. Phys. 65, 851-1112.

Golubitsky, M. \& Schaeffer, D. G. 1985 Singularities and Groups in Bifurcation Theory, I. Springer.

Golubitsky, M. \& Stewart, I. 2002 The Symmetry Perspective: From Equilbrium to Chaos in Phase Space and Physical Space. Birkhäuser.

Golubitsky, M., Stewart, I. \& Schaeffer, D. G. 1988 Singularities and Groups in Bifurcation Theory, II. Springer.

Guckenheimer, J. 1984 Multiple bifurcation problems of codimension two. SIAM J. Math. Anal. 15, 1-49. 
Iooss, G. \& Adelmeyer, M. 1998 Topics in Bifurcation Theory and Applications, 2nd edn. World Scientific.

Joseph, D. D. 1976 Stability of Fluid Motions I. Springer.

Julien, S., Lasheras, J. \& Chomaz, J.-M. 2003 Three-dimensional instability and vorticity patterns in the wake of a flat plate. J. Fluid Mech. 479, 155-189.

Lamb, J. S. W. \& Melbourne, I. 1999 Bifurcation from periodic solutions with spatiotemporal symmetry. In Pattern Formation in Continuous and Coupled Systems (ed. M. Golubitsky, D. Luss \& S. H. Strogatz), pp. 175-191. Springer.

Lamb, J. S. W., Melbourne, I. \& WulfF, C. 2003 Bifurcation from periodic solutions with spatiotemporal symmetry, including resonances and mode-interactions. J. Differential Equations 191, 377-407.

Lasheras, J. C. \& Meiburg, E. 1990 Three-dimensional vorticity modes in the wake of a flat plate. Phys. Fluids A 5, 371-380.

Lopez, J. M. \& MARques, F. 2003 Small aspect ratio Taylor-Couette flow: Birth of a verylow-frequency three-torus state. Phys. Rev. E 68, 036302.

Lopez, J. M., Marques, F. \& SAnchez, J. 2001 Oscillatory modes in an enclosed swirling flow. J. Fluid Mech. 439, 109-129.

Marques, F., Gelfgat, A. Y. \& Lopez, J. M. 2003 A tangent double Hopf bifurcation in a differentially rotating cylinder flow. Phys. Rev. E 68, 016310.

Marques, F., Lopez, J. M. \& Blackburn, H. M. 2004 Bifurcations in systems with $Z_{2}$ spatio-temporal and $O(2)$ spatial symmetry. Physica $D$ 189, 247-276.

Marques, F., Lopez, J. M. \& Shen, J. 2002 Mode interactions in an enclosed swirling flow: a double Hopf bifurcation between azimuthal wavenumbers 0 and 2. J. Fluid Mech. 455, 263-281.

Meiburg, E. \& Lasheras, J. C. 1988 Experimental and numerical investigation of the threedimensional transition in plane wakes. J. Fluid Mech. 190, 1-37.

Mullin, T. 1993 The Nature of Chaos. Oxford University Press.

Robichaux, J., Balachandar, S. \& Vanka, S. P. 1999 Three-dimensional Floquet instability of the wake of a square cylinder. Phys. Fluids 11, 560-578.

Rucklidge, A. M. 1997 Symmetry-breaking instabilities of convection in squares. Proc. Roy. Soc. Lond. A 453, 107-118.

Rucklidge, A. M. \& Silber, M. 1998 Bifurcations of periodic orbits with spatio-temporal symmetries. Nonlinearity 11, 1435-1455.

Sheard, G. J., Thompson, M. C. \& Hourigan, K. 2003 From spheres to circular cylinders: the stability and flow structures of bluff ring wakes. J. Fluid Mech. 492, 147-180.

Sheard, G. J., Thompson, M. C. \& Hourigan, K. 2004 From spheres to circular cylinders: non-axisymmetric transitions in the flow past rings. J. Fluid Mech. 506, 45-78.

Stevens, J. L., Lopez, J. M. \& Cantwell, B. J. 1999 Oscillatory flow states in an enclosed cylinder with a rotating endwall. J. Fluid Mech 389, 101-118.

Swift, J. W. \& Wiesenfeld, K. 1984 Suppression of period doubling in symmetric systems. Phys. Rev. Lett. 52, 705-708.

Tuckerman, L. S. \& Barkley, D. 2000 Bifurcation analysis for timesteppers. In Numerical Methods for Bifurcation Problems and Large-Scale Dynamical Systems (ed. E. Doedel \& L. S. Tuckerman), pp. 453-566. Springer.

Vogel, M. J., Hirsa, A. H. \& Lopez, J. M. 2003 Spatio-temporal dynamics of a periodically driven cavity flow. J. Fluid Mech. 478, 197-226.

Williamson, C. H. K. 1988 The existence of two stages in the transition to three-dimensionality of a circular cylinder wake. Phys. Fluids 31, 3165-3168.

Williamson, C. H. K. 1996 Three-dimensional wake transition. J. Fluid Mech. 328, 345-407.

WulfF, C., Lamb, J. S. W. \& Melbourne, I. 2001 Bifurcation from relative periodic solutions. Ergodic Theory and Dynamical Systems 21, 605-635. 